\title{
A new loading method for nanopaper and nanoparticles composite
}

\author{
Jin HuaYan ${ }^{1, a^{*}}$ and Ren Mei Xu ${ }^{2, b}$ \\ ${ }^{1}$ Guangdong Industry Technical College, China \\ ${ }^{2}$ Ball State University, USA \\ a Jhyan2000@yahoo.com, brxu@bsu.edu \\ * Corresponding author
}

Keywords: Nanopaper; Functional paper; Nanoparticles; In site reduction; Composite

\begin{abstract}
A new in site reduction method was introduced for nanopaper, typically for silver nanoparticles loading. Ag ions were spread into nanopaper, followed by immersion in $\mathrm{NaBH} 4$ solution. Ag-NPS were obtained successfully in the matrices without adding any other binders. The nanopaper micromorphology was characterized by SEM. AgNPS size was defined by TEM. Its binding force to substrate was assessed by FTIR. The thermal-stability was marked by TGA. This nanopaper showed superior properties for Ag-NPS loading. Nanopaper will have potential application in nanoparticles loading for functional paper field.
\end{abstract}

\section{Introduction}

Metal particles in the nanometer size range have attracted considerable interest in recent years, as they have many attractive applications in various fields [1-6] due to their unique size-dependent optical, electrical, magnetic, and antimicrobial properties. Silver nanoparticles (AgNPs) are believed to be size-dependent to their antimicrobial properties, where smaller AgNPs provide stronger antimicrobial effect than larger AgNPs [7]. The size of the nanoparticle implies that it has a large surface area to come in contact with bacterial cells and hence, it will have a higher percentage of interaction than bigger particles [8-10]. In this case, aggregation of AgNPs must be avoided because it will drastically decrease the accessibility of nanoparticles' surfaces, resulting in insufficient functionality. It is basically difficult to disperse metallic nanoparticles in a solvent, as nanoparticles tend to aggregate due to their high surface energy. Facile synthesis of morphologically controlled nanoparticles is a significant challenge in the field of nanotechnology.

Surface treatment is a way to load particles after matrices are formed. Nano paper was used as AgNPs load matrices in this study. AgNPs were reduced by reaction of silver ions. As a result, AgNPs were obtained successfully in the matrices without adding any other stabilizers. Micromorphology was characterized by SEM. AgNPs size was defined by TEM. Their binding force to paper was assessed by FTIR. The composite thermal-stability was marked by TGA. Regular filter paper was compared with the composite. Nanopaper will have potential applications in AgNPs loaded with nanoparticles stabilization and size control.

\section{Experimental part.}

Silver NPs (AgNPs) loading. Nanopaper is prepared in our lab. In situ loading of silver nanoparticles onto the nanopaper was carried out through the reduction of $\mathrm{AgNO} 3$ solution with varied concentrations $(10 \mathrm{mM}, 50 \mathrm{mM}, 100 \mathrm{mM}$, and $500 \mathrm{mM})$. Under ambient temperature $\left(\sim 25^{\circ} \mathrm{C}\right)$, several drops of AgNO3 solution were spread on the porous matrix, keeping totally wet for enough long time till the substrates were not absorbing solution. It is similar to that the substrates were soaked in AgNO3 solution for saturation and uniformity, but much less solution was used in this experiment.

After a certain time of air drying, a partially dehydrated matrix was obtained. It was then immersed in ethanol for $5 \mathrm{~min}$ and then in aqueous solution of NaBH4 $(50 \mathrm{mM})$ for $20 \mathrm{~min}$. Meanwhile, the 
color of the resultant samples turned to yellow or dark brown due to the reduction of Ag+ into silver nanoparticles. The composite was rinsed with Milli-Q water three times to remove water-soluble substances and free silver particles. Finally, the composite was dried in air.

Characterization. SEM nanopaper with/without AgNPs

The porous structures of nanopaper were examined using a field emission scanning electron microscope (FESEM) at an accelerating voltage of $20 \mathrm{kV}$ (a high resolution JEOL 6400F cold field emission SEM). The nanopaper with AgNPs were characterized using VPSEM (Variable Pressure Scanning Electron Microscope - Hitachi S3200N with an energy dispersive X-ray spectrometer). Energy-dispersive X-ray spectroscopic (EDS) analysis was also performed using the EDS detector connected with the VPSEM/FESEM with a voltage of $20 \mathrm{kV}$.

TEM preparation of nanopaper with AgNPs

A JEOL 2000FX transmission electron microscope (TEM) operating at $20.0 \mathrm{kV}$ was utilized to define the AgNPS in the nanopaper.

FTIR test. FT-IR spectroscopy was performed on a PerkinElmer Spectrum (Version 10.03.09). Spectra were obtained after the accumulation of 6 scans which had a resolution of $4 \mathrm{~cm}-1$ over the range of 4,000-650 cm-1.

TGA test. In order to determine the thermal decomposition temperature of composite films, thermogravimetric analysis (TGA) was used. It was operated on a Perkin Elmer TGA Q500 with a heating rate of $10^{\circ} \mathrm{C} / \mathrm{min}$ to $450^{\circ} \mathrm{C}$.

\section{Results and discussions}

Formation of AgNPs on Nanopaper. In this study, nanopaper was used as supports of AgNPs using the loading method above. With the increasing concentration of silver nitrate solution, the color turns darker. The process of AgNPs formation is the interaction between the micro/nano fibrillar cellulose matrix and Ag+. AgNO3 solution was absorbed by the matrix due to capillary force and cellulose's hydrophilic property, and silver ions diffused into the pores of the matrix and the surface of cellulose. Due to many hydroxyl groups in the cellulose structure, positive Ag+ could form complexes with $-\mathrm{OH}$ which holds a light negative charge. We suppose that the hydroxyl group is the place to anchor $\mathrm{Ag}+$, and then is the nucleus of $\mathrm{Ag}+$ to $\mathrm{Ag}(0)$ of silver crystals after addition of NaBH4 solution. The remaining silver ions were reduced to $\mathrm{Ag}$ and grew on the nucleus. Eventually AgNPs were formed under favorable reaction conditions. Nanopaper holds many more hydroxyl groups than regular cellulose, so these films should perform better than regular paper which is made of regular cellulose.

The regular paper is hardly observed with the silver particles under SEM no matter what AgNO3 concentration was used. Even $500 \mathrm{mM}$ AgNO3 solution was applied, the filter paper is monitored a few particles but aggregated in Figure.1. Paper sample EDS is analyzed to compare these three membranes in Fig.1. Filter paper shows the least amount of Ag. There are two reasons to explain the filter paper with fewer AgNPs. One is that the pore size is too large (tens of micrometer above) to keep the tiny AgNPs on the filter paper. The other is that the filter paper has much fewer hydroxyl groups than nanopaper which are the places to anchor $\mathrm{Ag}+$.

TEM for characterization of AgNPs formed on nanopaper

The formation of AgNPs into nanopaper was confirmed by TEM observation (Figure.2). The existence of AgNPs can be seen clearly in the films. For the paper, we also found a lot of particles dispersed well while we hardly found them in SEM, though the paper composite is changed color to, such as yellow, brown, etc. and containing impurities for sure. It implies that AgNPs are not located on the surface but inner paper. This kind of paper composite may be beneficial in applications since it still holds paper properties as information media, like writing and printing. We don't discuss it here. 

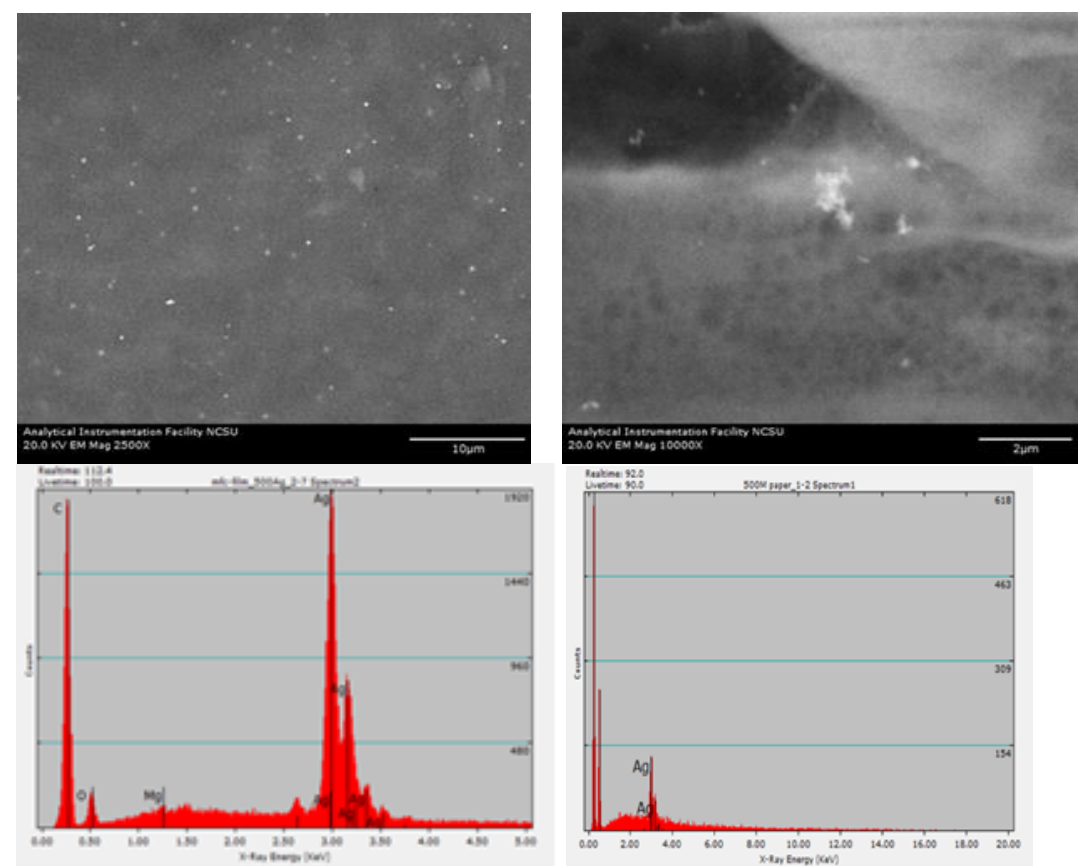

Figure. 1 SEM of Nanopaper-Ag (left) and regular paper-Ag (right) and their EDS
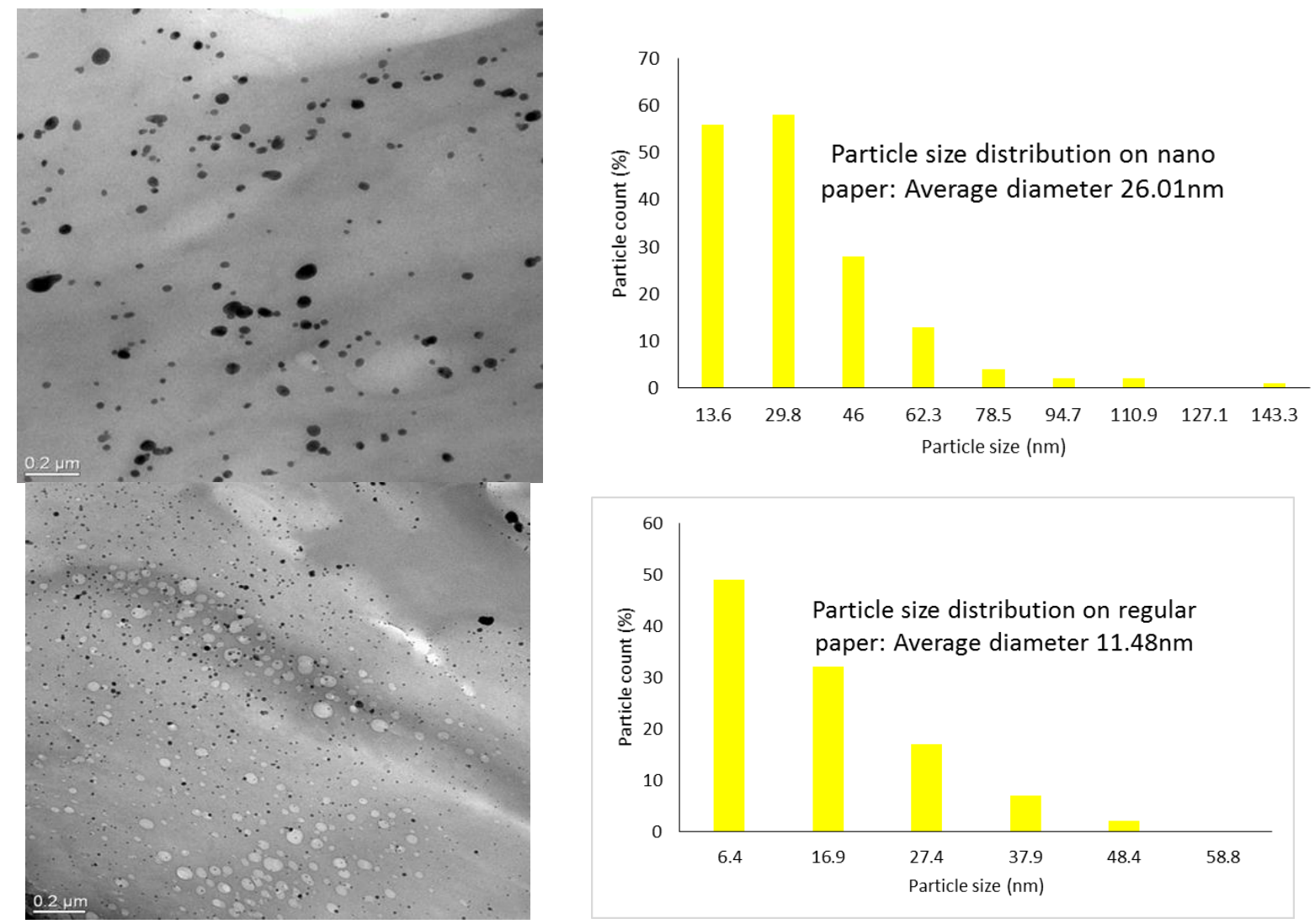

Figure. 2 TEM of AgNPS loaded nanopaper(up) and Regular Paper(down) and its particles size distribution

In order to define the size of nanoparticles and its distribution, ImageJ software is used. The analyzed results of TEM images are obtained and also showed in Fig.2. The particle size on nanopaper is obviously larger than on regular paper. The size of most of the AgNPs on nanopaper is in the range of 10-30 $\mathrm{nm}$ which accounts for more than $60 \%$ particles. The size of AgNPs on paper is much smaller which $70 \%$ above is less than $15 \mathrm{~nm}, 45 \%$ of AgNPs particles is less than 10nm. Both 
nanopaper and paper can be good supports for mono dispersed AgNPs composite. But it exist an optimum AgNPs size to reach the best antibacterial performance[11].

FTIR test results. The spectra of nanopaper with and without AgNPs are shown in Figure.3. The two substrates display the characteristic bands of cellulosic fibrils at $3360 \mathrm{~cm}-1,2918 \mathrm{~cm}-1,1028$ cm-1 for $\mathrm{OH}, \mathrm{CH}$, and C-C. No significant changes are observed for two nanopapers, and even regular paper, except two more bands at 1600-1700 cm-1 and $896 \mathrm{~cm}-1$ of nanopaper for carbonate group due to oxidized treatment. After AgNPs loading, the nanopapers showed some peak weakening with their spectra, typically at $3360 \mathrm{~cm}-1$. There is an obviously lower peak than blank films. This is due to the tight absorption of Ag into the cellulose hydroxyl group, and there is no chemical reaction since the band is not changed. On the other hand, the Ag-paper keeps the almost exact same shape as its blank, indicating little absorption of Ag into the regular paper surface. This also explained why we couldn't observe the AgNPs on regular paper under SEM.

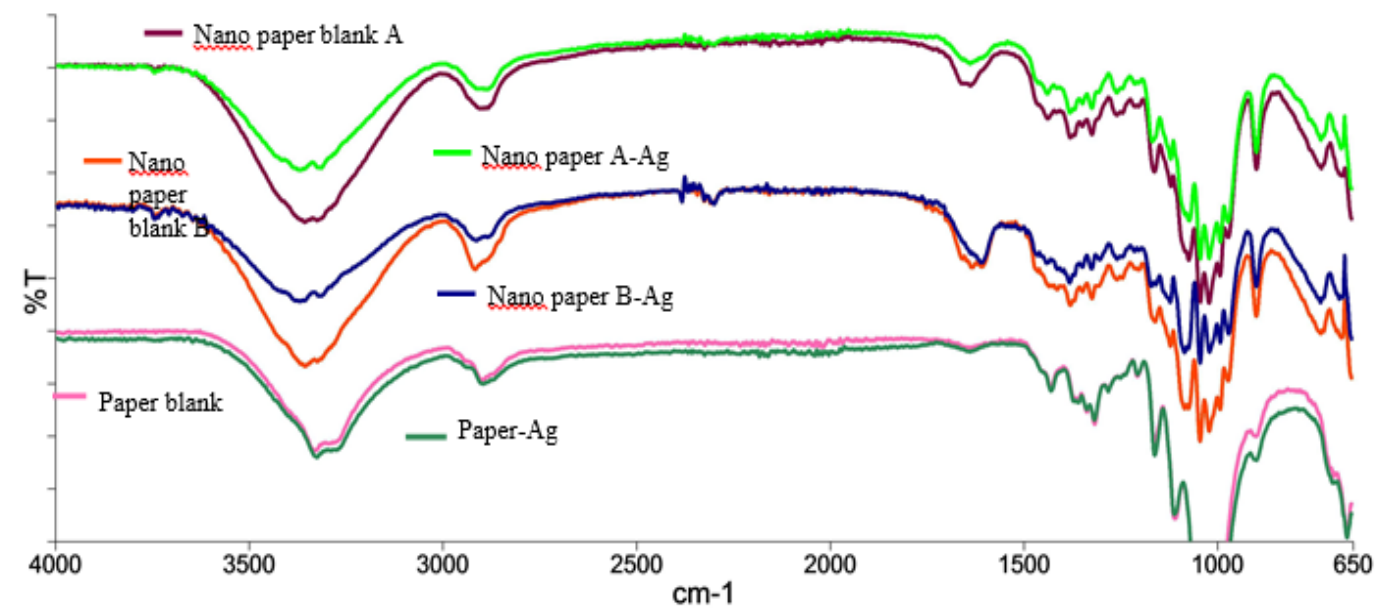

Fig. 3 IR spectra of three blank membranes and their Ag loaded membranes (treated with $50 \mathrm{mM}$ $\mathrm{AgNO}_{3}$ )

TGA test results. In view of the importance of thermal stability in many applications of AgNPs nanopaper, we examined thermal decomposition of metal-cellulose by thermogravimetry (TGA) in a nitrogen atmosphere, as shown in Fig.4. In all TGA curves, the small weight losses below $150^{\circ} \mathrm{C}$ apparently resulted from evaporation of adsorbed moisture. Under nitrogen, the decomposition behavior of the nanopaper-Ag was nearly the same with different levels of $\mathrm{Ag}$, the most weight loss taking place at $250^{\circ} \mathrm{C}$. The presence of AgNPs improved the thermal stability since the blank nanopaper decomposition temperature is around $288^{\circ} \mathrm{C}$, but the AgNPs-nanopaper decomposition temperatures are $308^{\circ} \mathrm{C}$. The result is similar with the regular paper. The paper decomposition $\mathrm{T}$ is $332^{\circ} \mathrm{C}$, but with the presence of AgNPs, the paper composites had $10^{\circ} \mathrm{C}$ increase in decomposition temperature to around $342^{\circ} \mathrm{C}$. 


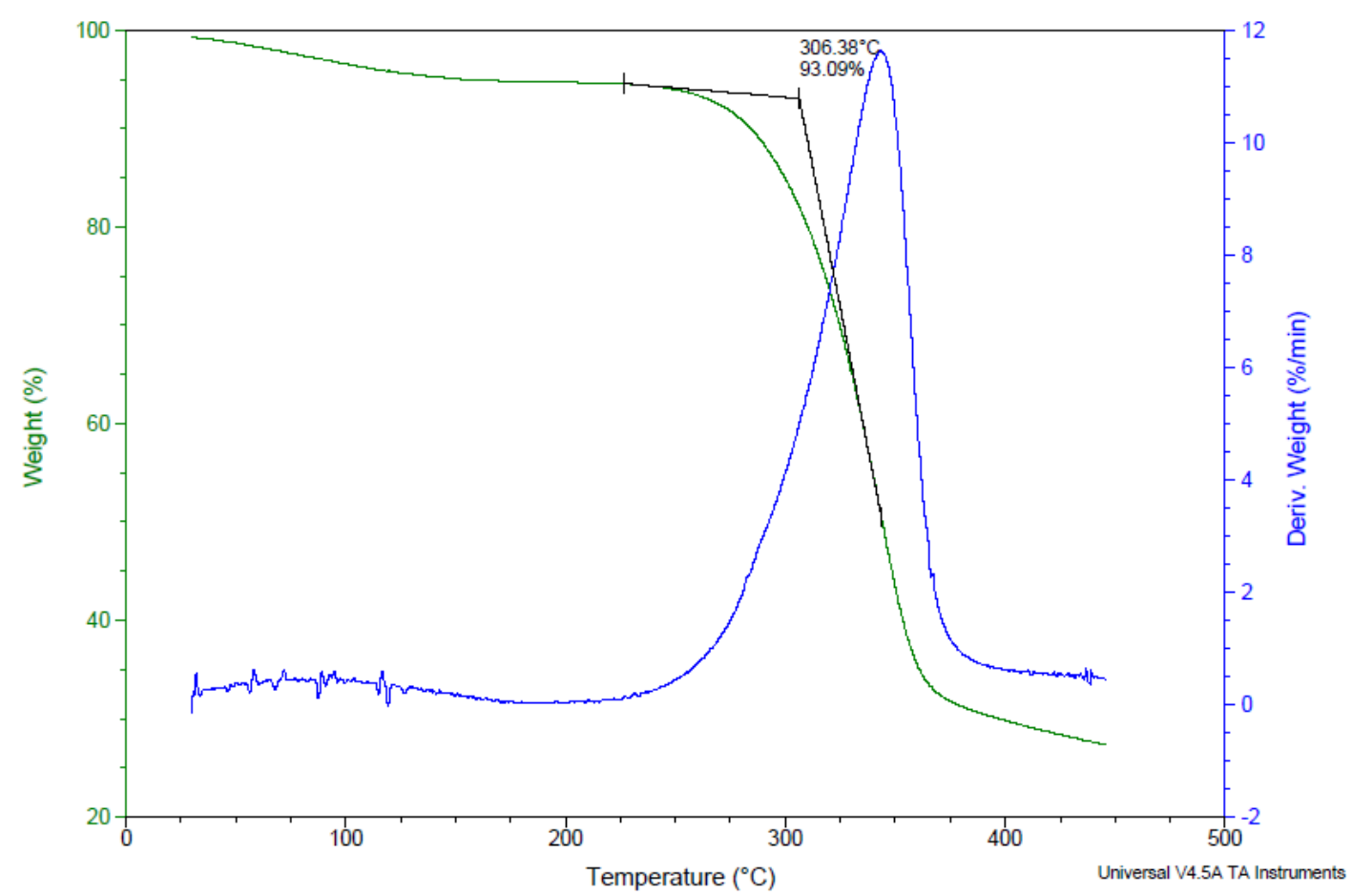

Fig. 4 TGA of AgNPs loaded nanopaper treated by 100mM AgNO3 solution

It is considered that AgNPs in nanopaper induced certain interaction with cellulose in molecular level. During $\mathrm{Ag}+$ to $\mathrm{Ag}(0)$ reduction, nanopaper rich hydroxyl groups are the places to anchor $\mathrm{Ag}+$. With proper pore size control, well dispersed AgNPs help the composite stability.

\section{Conclusion}

In this work we have shown that nanopaper can be facilely used as substrate for preparation and stabilization of nanoparticles of AgNPs. AgNPs are well dispersed on the film with a range diameter of 10-30nm. The nanopaper hybrid films performed strong antibacterial properties. We therefore propose that the procedure described here, the metal ions are anchoring on the nanopaper due to their negative charged groups, to first perform chemical reactions as the core, and then grow particles, will provide a technically more feasible procedure. The work is also a demonstration that highly fibrillated nanopaper shows desirable properties that lead to new methods in technology.

\section{References}

[1] Z. Shervani, Y. Ikushima, M. Sato, H. Kawanami, Y. Hakuta, T. Yokoyama, T.Nagase, H. Kuneida, K. Aramaki, Colloid Polym. Sci. 286 (2008) 403.

[2] C. Chen, L. Wang, H. Yu, J. Wang, J. Zhou, Q. Tan, L. Deng, Nanotechnology 18 (2007) 115612.

[3] Zhong C and Maye M M, Core-Shell Assembled Nanoparticles as Catalysts, 2001 Adv. Mater. 13 1507

[4] Kim Y, Johnson R C and Hupp J T, Gold Nanoparticle-Based Sensing of 'Spectroscopically Silent" Heavy Metal Ions, 2001 Nano Lett. 1165

[5] J. Zhu, S. Liu, O. Palchik, Y. Koltypin, A. Gedanken, Shape-controlled synthesis of silver nanoparticles by pulse sonoelectrochemical methods, Langmuir 16 (2000) 6396.

[6] M. Li, H. Schnablegger, S. Mann, Coupled synthesis and self-assembly of nanoparticles to give structures with controlled organization, Nature 402 (1999) 393. 
[7]Mahndra Rai, Alka Yadav, Aniket Gade, Silver nanoparticles as a new generation of antimicrogials, Biotechnology Advances 27(2009) 76-83

[8] Morones JR, Elechiguerra JL, Camacho A, Ramirez JT. The bactericidal effect of silver nanoparticles. Nanotechnology 2005; 16:2346-53

[9] Raimondi F, Scherer GG, Kotz R, Wokaun A. Nanoparticles in energy technology: examples from electochemistry and catalysis. Angew. Chem., Int. Ed. 2005; 44:2190-209.

[10] Pal S, Tak YK, Song JM. Does the antibacterial activity of silver nanoparticles depend on the shape of the nanoparticle? A study of the gram-negative bacterium Escherichia coli. Appl Environ Microbiol 2007; 27(6):1712-20.

[11] A. Panacek, L. Kvitek, R. Prucek, etc., ,Silver Colloid Nanoparticles: Synthesis, Characterization, and Their Antibacterial Activity, J. Phys. Chem. B 2006, 110, 16248-16253 